\title{
Swimmy Disease Diagnosis Based on PLC Control System Design and Implementation
}

\author{
Changzheng Li, Zhiyi Qu, and Yong Zhao
}

\begin{abstract}
PLC (Programmable Logic Controller) is a type of electric control device, as compared with the traditional control devices, it has many advantages, so PLC is used in industy widely. Vertigo is an illusion of motion, as the surrounding environment or its rotation, or swing instability, shaking, top-heavy sense. The clinical manifestation is the uncontrolled eyes nystagmus. Based on the symptoms, we can assess the presence of disease by eye movements of people in different positions.

This paper focuses on the design and realization of control system based on PLC vertigo disease diagnosis, the whole system includes rotating equipment and PC. Rotating equipment mainly includes electrical equipment, mechanical equipment, PLC, etc. The PC part is the software part, including the PLC program, PLC interface program and the main control program. Proved by the experiment, the control equipment has met the predetermined technical requirements. Achieving the requirements of clockwise rotation, counter clockwise rotation, continuous rotation, rotation of single or several rings. Compared with other similar diagnostic equipment, the operation is simple safe and reliable. The patient is positioned accurate, so the diagnosis process is rigorous, providing the reliable diagnostic basis for diagnosis of vertigo disease.
\end{abstract}

Index Terms-PLC, diagnosis of vertigo, rotation, communication.

\section{INTRODUCTION}

With the progress of science, the industrial control technology has been developed rapidly, especially in recent years, the industrial control technology has been widely used in petroleum, chemical industry, machinery manufacturing, education, medical and other fields, it has important application in logic control of switching value, analog quantity control, motion control, process control, data processing communication etc. PLC plays an irreplaceable role in modern industrial control technology. In this paper, the rotation of large vertigo diagnosis device is controlled by PLC, to achieve a predetermined angle, thus provide Auxiliary diagnosis to vertigo disease. This paper elaborates the important application of PLC in the field of medicine with specific examples.

\section{APPLICATION BACKGROUND}

Programmable logic controller, referred to as PLC, is a kind of automation device with microprocessor as the core,

Manuscript received May 13, 2014; revised August 30, 2014.

The authors are with the College of Information Science and Engineering of Lanzhou University, Lanzhou, China (e-mail: 1585239867@qq.com; quzy@1zu.edu.cn; fm2zhaoyong@163.com). a kind of electronic system with digital operation used in the working environment, it can use the directives Stored in the internal of PLC, which was designed by users to achieve the logical calculus, sequential operation, timing and arithmetic, and through digital or analog I/O to control various types of machinery or process [1].

Vertigo is an optokinetic illusion or position of illusion produced by the disorder of spatial positioning, the disease is caused by many reasons, so the vertigo disease is generally difficult to give a definition, and it cannot be measured [2]. In addition, most of the vertigo patients are not sure about the symptoms or they can't describe the symptom accurately. We only over emphasize it, or identify it according to our subject feelings. As a result, diagnosis of vertigo disease error is large, the misdiagnosis rate is also high, and it has brought the huge challenge for the diagnosis of the disease. One of the most important clinical manifestations is the uncontrolled eyes nystagmus will happen to a man if he is in different postures. We can assess the presence of disease through the human eye movement in different positions based on the causes and symptoms of vertigo.

Based on the above introduction, this paper main tells processes to carry out an effective diagnosis of vertigo disease: Through the host computer to operate PLC, then control the rotating equipment rotating horizontal, vertical rotation, clockwise rotation, counterclockwise rotation, single turn, multi turn, and continuous rotation, finally reach the specified location, then observed the eye movement.

\section{CONTROL SYSTEM}

The control system consists of a computer; connect computer and PLC, PLC of three parts. Among then, use the RS-232 interface to connect connection line. Connection diagram is shown in Fig. 1 [3].
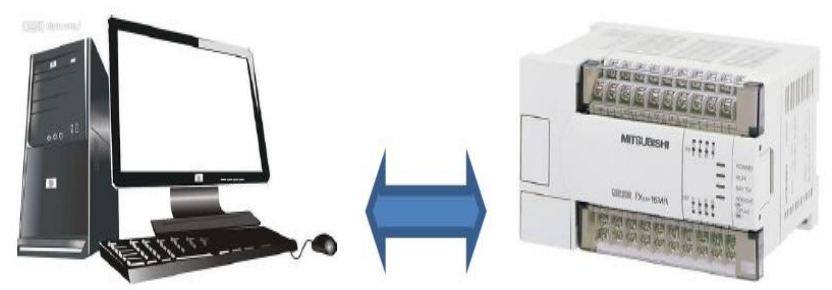

Fig. 1. The system block diagram

\section{A. $P L C$}

The system uses mitsubishi PLC (Mitsubish Programmable Logic Controller Mitsubishi), the product model is the FX2N series, the series of PLC is small, high 
speed, high performance, in addition to FX series PLC generic can independently use 16-25 input and output point, it also can be applied to multiple base connection between components, logic operation, analog control, timing, location, counting and other special purpose, even if users do not know the other side of the hardware and communication protocol, it can also be used for communication. It is a set of PLC which can meet the diverse and extensive requirements. Its Characteristics can be summarized as follows:

1) The system configuration is fixed and flexible;

2) Programming is simple;

3) Variety is rich, and can be chosen freely;

4) Reassuring high performance;

5) High speed computing;

6) Used in a variety of special purpose;

7) External machine communication is simplistic;

8) Common external devices.

\section{B. $R S-232$}

RS-232 is one of the interfaces of the computer and other communication devices, which is composed by EIA (Electronic Industries Association) of asynchronous transmission standard interface, RS - 232 interface usually has 9 pins, as shown in Fig. 2 for the RS-232 interface diagram.

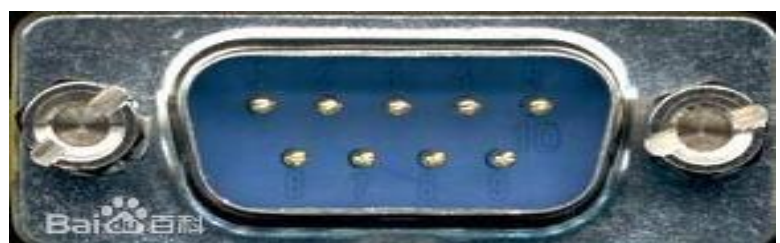

Fig. 2. RS - 232 interface diagram, the upper left corner is 1, the lower right corner is 9 .

Each pin is described as shown in Table I [4].

\begin{tabular}{|c|c|c|c|c|c|}
\hline Pin (9 pins) & Pin (25 pins) & Signal & $\begin{array}{l}\text { Signal } \\
\text { source }\end{array}$ & Type & Description \\
\hline 1 & 8 & $\mathrm{CD}$ & DCE & control & $\begin{array}{l}\text { Data carrier detects this pin. It can be controlled by } \\
\text { the Modcm, when the phone is switched on, the } \\
\text { Modcm is using this pin to inform the computer the } \\
\text { detected the carrier, which is online }\end{array}$ \\
\hline 2 & 3 & $\mathrm{RD}$ & DCE & data & Receiving remote serial data \\
\hline 3 & 2 & TD & DTE & data & Sending out the serial data of the computer \\
\hline 4 & 20 & DTR & DTE & control & $\begin{array}{l}\text { This pin is controlled by the computer, when it is of } \\
\text { high potential, it can transmit data }\end{array}$ \\
\hline 5 & 7 & GND & & & $\begin{array}{l}\text { Grounding terminal. Signal place and signal line in } \\
\text { protecting area }\end{array}$ \\
\hline 6 & 6 & DSR & DCE & control & $\begin{array}{l}\text { Ready for data equipment. This pin can be controlled } \\
\text { by Modcm, when it is of high potential, Modcm will } \\
\text { tell computer to be ready, then you can send the data }\end{array}$ \\
\hline 7 & 4 & RTS & DTE & control & $\begin{array}{l}\text { A request to send. This pin is controlled by the } \\
\text { computer, it shows that DTE requests DCE to send } \\
\text { out data, when it is of high potential, the computer } \\
\text { requests Modcm to send out data }\end{array}$ \\
\hline 8 & 5 & CTS & DTE & control & $\begin{array}{l}\text { Clear to send. This pin can be controlled by Modcm, it } \\
\text { is used to represent the DCE which is ready to receive } \\
\text { the data sent by DTE, It is also a response to a request } \\
\text { signal transmitted signal RTS }\end{array}$ \\
\hline 9 & 22 & RI & DCE & control & $\begin{array}{l}\text { Ringing detection. If there is a phone call, the pin can } \\
\text { be detected by the Modcm, then inform the computer } \\
\text { whether you want to answer the call }\end{array}$ \\
\hline
\end{tabular}

RS232 provides a process of connecting cable and mechanical, electrical properties, signal function and transmission. The latest version is TIA-232-F issued by America Telecommunications Industry Association (an organization classified by EIA). It is also USA national standard ANSI/TIA-232-F-1997 (R2002), this standard was reconfirmed in 2002. The numbers TIA/EIA-232-F and ANSI/TIA/EIA-232-F-1997 were released by TIA/EIA in 1997 while the previous version was TIA/EIA-232-E [5].

\section{COMmunicAtion BETWEen THE CONTROL Systems}

This system uses Visual Studio as a development platform, using $\mathrm{C \#}$ as the programming language, using Mitsubishi MX components, provides a communication method between industrial controlled computer and PLC of Mitsubishi FX2N Series [6]. This system realized the accurate positioning vertigo rotation of rotating equipment according to the method; the practical application effect is good. Transmission rate of the communication system is $9600 \mathrm{bps}$, the set port is com1. The communication of different parts of the structure is shown in Fig. 3.

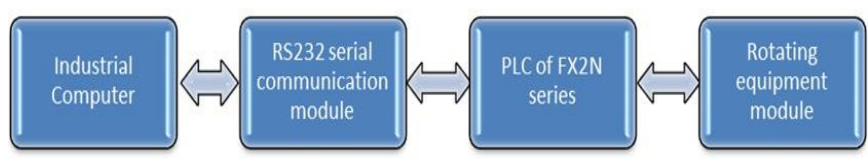

Fig. 3. Communication structure.

\section{A. Hardware Setup}

\section{1) Set of Mitsubishi MX components}

Mitsubishi MX communication software package is a developed auxiliary means corresponding to the Windows system software; it can easily establish communication 
between computer and Mitsubishi controller products, and realize data monitoring. The software package can make the design of the application in $\mathrm{VC}++, \mathrm{C}, \mathrm{VB}$ simple. The software package can achieve communication between various communication networks and control interface without involving any communication protocols. MX communication software package is a Software-aided design tool designed by Mitsubishi to enhance the second development capabilities of its FA (Factory Automation) products. At present, the MX communication software package to connect communication PLC including Mitsubishi Q, QnA, A / AnS and FX series in the network, including Ethernet, MELSECNET / H, CC link or RS-232 serial communication module. All communication path to the MX component to support the PLC and computer, communicate by simply setting the can, so the development efficiency of the system has been greatly improved.

The MX module contains many DLL; each DLL corresponds to different ACT Controls, the PLC can be accessed by calling functions in ACT Controls. In this paper, ActEasyIF Control in ActMultidll is used as a communication in order to simplify the communication settings in the communication setting utility, can communicate via any communication path.

First to introduce the ActMulti.dll dynamic link library in engineering project, and then set ActEasyIF correctly, attributes and the default values of the ActEasyIF control as shown in Table II.

TABLE II: ACTEASYIF CONTROLS THE ATTRIBUTES AND THE DEFAULT

\begin{tabular}{|l|l|l|}
\hline Attribute Name & $\begin{array}{l}\text { Default } \\
\text { Value }\end{array}$ & Attribute Specifications \\
\hline $\begin{array}{l}\text { ActLogicalStati } \\
\text { onNumber }\end{array}$ & 0 & $\begin{array}{l}\text { Logical station number Set in the } \\
\text { communication settings utility }\end{array}$ \\
\hline ActPassword*1 & Empty & $\begin{array}{l}\text { Passwords set in the connected station } \\
\text { side A6TEL, Q6TEL, Compatible } \\
\text { Module C24 of Q series, and } \\
\text { Compatible Module E71 of Q series. }\end{array}$ \\
\hline
\end{tabular}

\section{2) PLC setting}

PLC is designed to make communication between devices, only set to be consistent, can get reliable communication between devices. In this paper, the parameters are configured through the PLC programming software (GX developer). The parameter configurations are shown in Fig. 4.

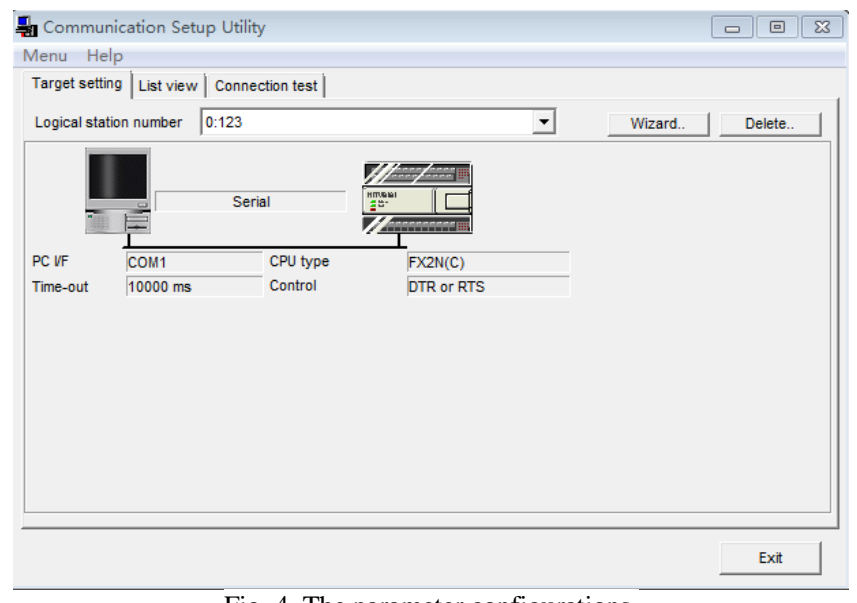

Fig. 4. The parameter configurations.

\section{B. Communication Programs}

The computer as the upper machine, development platform for Microsoft Visual Studio 2012 integrated development environment, development language is c \#, MX Component components issued by mitsubishi, by calling the ActEasyIF control to realize the communication between computer and PLC [7]. Communication program flow diagram is shown in Fig. 5.

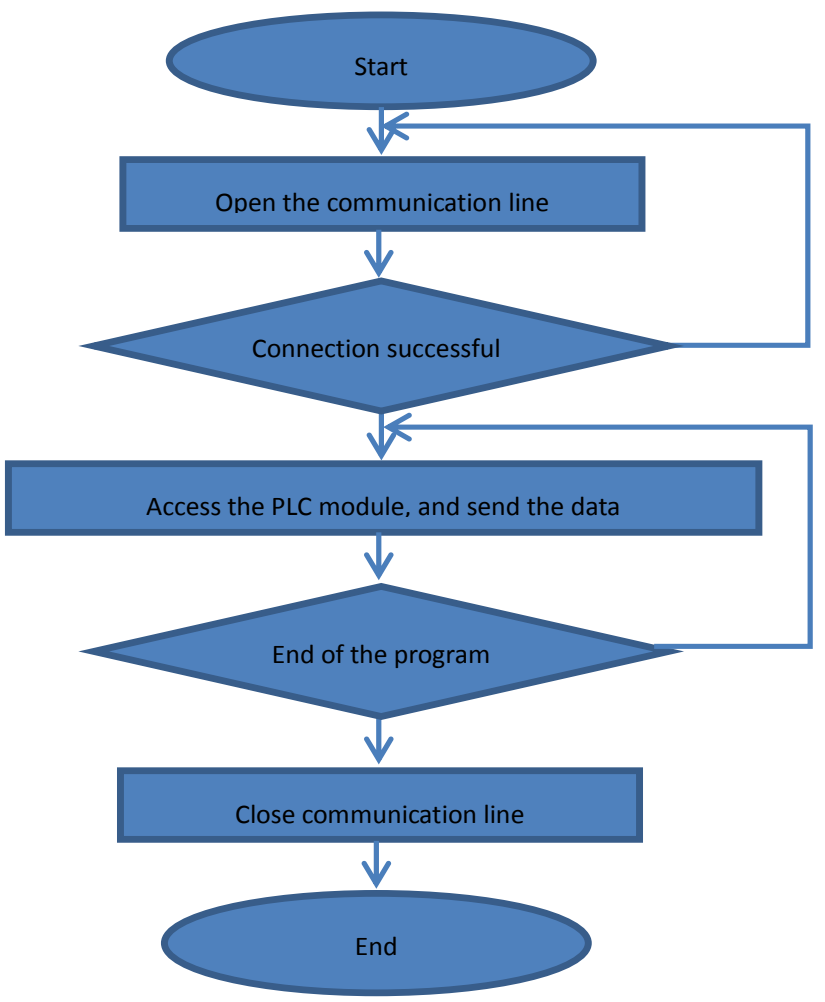

Fig. 5. Communications program flow chart.

\section{1) Initialization $M X$ control}

According to the description in Table I, attributes and default values of ActEasyIF control should be set firstly. The corresponding C\# language codes are:

\section{ActEasyIF1.ActLogicalStationNumber $=1$;}

$$
\text { ActEasyIF1.ActPassword = null; }
$$

\section{2) PLC Read-write operation}

Open PLC connection is using Open function of ActEasyIF.

The read operations function to PLC involves:

1) ReadDeviceBlock

2) ReadDeviceRandom

3) GetDevice

Among them, option A is suitable for the batch reading soft component data. Option random reading soft component element, Option $\mathrm{C}$ is suitable to get data from a spot of the soft component.

The write operations function to PLC involves:

1) WriteDeviceBlock

2) WriteDeviceRandom

3) SetDevice

Among them, option A is suitable for the batch writing data to the soft element. Option B is suitable for writing 
random data to the soft component, Option $\mathrm{C}$ is suitable to set a spot of the soft component

After having finished operating ActEasyIF, we should use the "Close" function to close PLC.

In this system, the data read from the soft components and write soft component data for the corresponding equipment should be rotation Angle value.

In addition, in the process of communication, don't disconnect or reconnect the RS232 cable frequently, don't reset the CPU of the PLC frequently or open/close the power of CPU in PLC, otherwise may cause unrecoverable communication error. If such a communication error exists, we should cut the Rs232 cable completely, and wait for more than 5 seconds, then reconnect it.

\section{THE EXPERIMENTAL RESULTS}

The interface realized the communication part of this system is shown in Fig. 6. The red arrow location shows the rotating angle set by rotating equipment, the speed indicator shows the rotating speed set by the rotating equipment, and these data are written to the soft components in the respectively. The intermediate character model indicates position of the rotating equipment; the data are output from the corresponding soft components separately. Proved by experiments, rotating equipment can be preset rotating in accordance with the instructions sent by computer, and the rotary position is accurate.

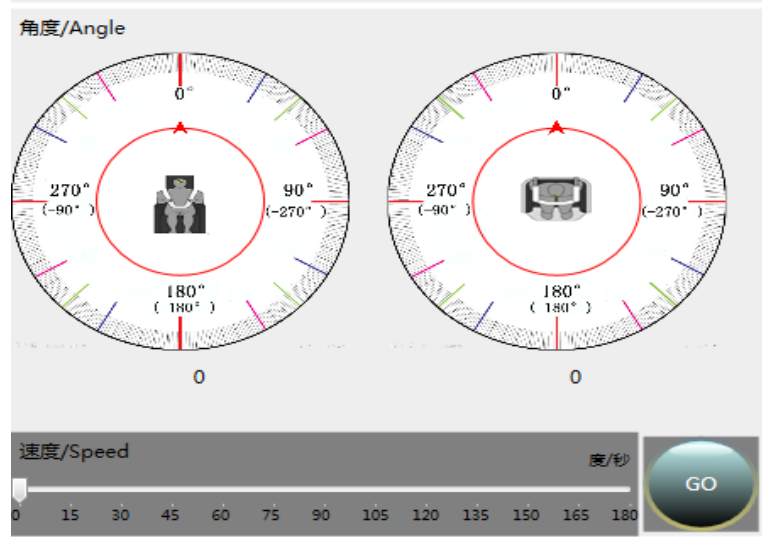

Fig. 6. The interface realized the communication part of this system.

\section{CONCLUSION}

Modern industrial control system can simplify the communication design greatly by using means of communication between MX components and the host computer, thus improve the developing efficiency to a great extent, and help developers to save writing cumbersome codes in communication part, and further improves the stability, reliability and flexibility of the system, realizes the purpose that common control configuration software can not achieve, the actual application effect is good. In addition, the method can be extended to other PLC series of Mitsubishi, it has scalability and portability.

\section{REFERENCES}

[1] W. Tan, "The design and implementation of industrial control system based on PLC."

[2] J. R. Tian, "Advances in diagnosis and treatment of vertigo," Chin J Geriatr Heart Brain Vessel Dis, vol. 14, no. 12, p. 1335, Dec 2012.

[3] C. Liang, "The communication between PLC and PC man-machine interface based on VB."

[4] L. J. Ge, "The realization of RS232 serial interface communication based on C language," Journal of Hebei University of Technology, no. 6, December 2008

[5] The Interface between the Data Terminal Equipment and Data Circuit-Terminating Equipment Employing Serial Binary Data Interchange, TIA Standard, TIA-232-F, ANSI/TIA-232-F-1997.

[6] S. P. Luan, "Design of SCADA based on C\# and Mitsubishi MX component," p. 38

[7] Z. G. Yan, T. M. Shen, and J. Jin, "Application of PLC VB serial communication in the back scattered human tester based on motion control," Police Technology, pp. 61-64, September 2010.

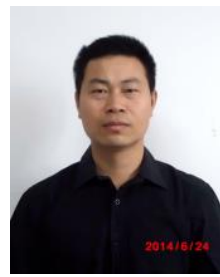

Changzheng Li was born in Changsha, Hunan, China on January 4, 1978. He is a postgraduate student in the College of Information Science and Engineering of Lanzhou University, majoring in computer application technology.

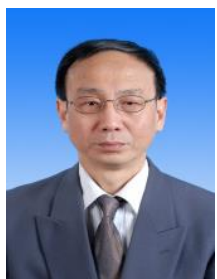

Zhiyi Qu was born in Lanzhou, Gansu, China in 1957. He is working as a professor in the LanZhou University School of Information Science \& Engineering, and his study areas are multimedia processing and communication technology.

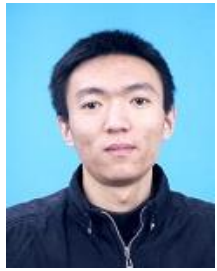

Yong Zhao was born in Xingtai, Hebei, China in 1987. He is a graduate student studying C and majoring in computer application technology. 